\title{
Production of Bacteriocin E50-52 by Small Ubiquitin-Related Modifier Fusion in Escherichia coli
}

\author{
QING WANG ${ }^{1 *}$, WENJUAN FU ${ }^{1 *}$, QINGSHAN MA ${ }^{1}$, ZHANQIAO YU $^{1}$ and RIJUN ZHANG \\ ${ }^{1}$ Laboratory of Feed Biotechnology, State Key Laboratory of Animal Nutrition \\ College of Animal Science \& Technology, China Agricultural University, Beijing 100193, People’s Republic of China \\ Submitted 8 January 2013, revised 6 April 2013, accepted 18 October 2013
}

\begin{abstract}
Bacteriocin E50-52, a class IIa bacteriocin with a wide antibacterial spectrum, and has a huge potential to be a substitute for conventional antibiotics. In this research, the bacteriocin E50-52 gene was cloned into the expression vector pET SUMO (small ubiquitin-related modifier) and introduced into Escherichia coli BL21 (DE3). The recombinant fusion protein SUMO-bacteriocin E50-52 expressed in a soluble form was purified to a purity of more than $90 \%$ by Ni-NTA sepharose column and $117 \mathrm{mg}$ fusion protein was obtained per liter of fermentation culture. The fusion protein was cleaved with SUMO protease and re-applied to a Ni-NTA Sepharose column. Finally, about 16 mg recombinant bacteriocin E50-52 (rbE50-52) was obtained from a 1-liter fermentation culture with no less than 95\% purity. The rbE50-52 had similar antimicrobial properties and molecular weight as the native bacteriocin E50-52 and showed very low hemolytic activity.
\end{abstract}

Ke y words: Escherichia coli, bacteriocin E50-52, purification, recombinant expression, small ubiquitin-related modifier

\section{Introduction}

Bacteriocins are a family of ribosomally synthesized antimicrobial peptides produced by bacteria (Klaenhammer, 1993; Cleveland et al., 2001). Studies suggest that bacteriocins can inhibit bacteria by forming pores in the membrane and have effective activity against antibiotic-resistant pathogens (Jenssen et al., 2006; Svetoch and Stern, 2010). There is no report showing that bacteriocins could be toxic for animals and accumulate in the treated subjects (Svetoch and Stern, 2010). Therefore, as promising new biological products, bacteriocins could be developed into novel alternatives to traditional antibiotics (Joerger, 2003). Bacteriocin E50-52 is a class IIa bacteriocin produced by Enterococcus faecium NRRL B-30746 and has a molecular weight of 3339.7 Da. Bacteriocin E50-52 showed potent activity against both Gram-positive and Gram-negative bacteria in vitro tests and therapeutic trials. Moreover, bacteriocin E50-52 is heat-stable and can inhibit drugresistant nosocomial bacteria effectively (Svetoch et al., 2008 ; 2009). Thus, bacteriocin E50-52 could be considered as a potential candidate for antibacterial therapy.

It is often cumbersome, time-consuming, and uneconomical to isolate bacteriocins from natural producer strains. Though chemical synthesis is a good way to obtain peptides of interest, it is limited by expensive reagents. Heterologous expression in E. coli, the first host used to produce a recombinant pharmaceutical peptides, is an adaptable method for the economical and rapid production of target proteins (Swartz, 2001; Li et al., 2007; Jasniewski et al., 2008). However, the significant antimicrobial activity of bacteriocins poses difficulties for direct expression in the host and high susceptibility to proteolytic degradation decreases the efficiency of purification (Li et al., 2009). For these reasons, the potential fusion expression strategy is employed to protect E.coli and target peptide (Rao et al., 2004; Wei et al., 2005; Morin et al., 2006; Shen et al., 2010; Ma et al., 2012).

SUMO is a member of the ubiquitin protein family that modulates the properties of intracellular proteins (Muller et al., 2001; Johnson, 2004). When fused with proteins, SUMO can fold and protect them via its chaperoning functions (Li et al., 2010). As a new fusion system, SUMO appears to have all the advantages of traditional systems and can be cleaved without extension in the $\mathrm{N}$-terminus of the target proteins (Butt et al., 2005). Therefore, in this study, SUMO fusion protein expression system was used to produce rbE50-52.

\footnotetext{
* Corresponding author: R. Zhang, No. 2 Yuanmingyuan West Road, Haidian District, Beijing 100193, China; phone: + 8610-62731208; fax: + 8610-87969550; e-mail: feedbiotech@yahoo.com

* These authors contributed equally to this work.
} 


\section{Experimental}

\section{Materials and Methods}

Bacterial strains and growth conditions. E. coli bacterial strain Mach $1^{\mathrm{TM}}-\mathrm{T}^{\mathrm{R}}$ and strain BL21 (DE3) (Invitrogen, USA) were used as subcloning and expression host, respectively. Both E. coli Mach $1^{\mathrm{TM}}-\mathrm{T} 1^{\mathrm{R}}$ cells and BL21 (DE3) cells were cultured and selected in LB broth or agar (1.8\%) supplemented with kanamycin $(50 \mu \mathrm{g} / \mathrm{ml})$ at $37^{\circ} \mathrm{C}$. Staphylococcus aureu ATCC 25923 was purchased from the China General Microbiological Culture Collection Center (Beijing, China), E. coli K88 and Listeria monocytogenes IVDCC 53005 were purchased from the China Veterinary Culture Collection Center (Beijing, China).

Plasmid and gene sequence. pET SUMO (Invitrogen) was used as expression plasmid. The GenBank accession number for the bacteriocin E50-52 used in this study is $\underline{P 85148}$.

Construction of expression vectors. The bacteriocin E50-52 gene was synthesized (Invitrogen) and amplified using primer pairs (Primer E50-52 F: 5'-ACCACCAAAAACTATGGCAAC-3', Primer R: 5'-TCACTATTACGCCAGTTTGCACAG-3'). The resulting PCR product was separated by $1.8 \%$ gel electrophoresis, purified using a DNA gel extraction kit (Tiangen), and ligated into the linearized pET SUMO plasmid by TA cloning. The ligation mixture was transformed into E. coli Mach $1^{\mathrm{TM}}-\mathrm{T} 1^{\mathrm{R}}$ cells for propagation of the recombinant plasmid. Another PCR was performed using primer pairs (Primer SUMO F: 5'-AGATTCTTGTACGCAGGTATTAG-3'; Primer T7 R: 5'-TAGTTATTGCTCAGCGGTGG-3'), and the product containing inserted fragment was sequenced to confirm insert orientation and sequence fidelity.

Expression of rbE50-52 fusion protein. The constructed pET SUMO-bacteriocin E50-52 plasmid was transformed into E. coli BL21 (DE3) strain. The recombinant expression strain was cultured in LB broth containing kanamycin at $37^{\circ} \mathrm{C}$ with shaking $(180 \mathrm{rpm})$ to an optical density $\left(\mathrm{OD}_{600}\right)$ of $0.7-0.8$. Isopropyl $\beta-\mathrm{D}-1$ thiogalactopyranoside (IPTG) was then added to a final concentration of $1.5 \mathrm{mM}$ to induce the expression of the recombinant protein at $37^{\circ} \mathrm{C}$ for $5 \mathrm{~h}$. To obtain the recombinant protein, the cells were treated according to the manufacturer's instruction. The disrupted cells were centrifuged at $13,000 \mathrm{~g}$ for $2 \mathrm{~min}$ to separate the soluble supernatant and the insoluble pellet fraction. Both supernatant and precipitate were analyzed by Tricine-SDS-PAGE. The non-induced cells were used as negative control.

Purification of fusion protein. The solubilized SUMO-bacteriocin E50-52 fusion protein was applied to a pre-equilibrated Ni-NTA (GE, USA) Sepharose column $(5 \mathrm{ml})$, and the bound protein was eluted by elution buffer $\left(50 \mathrm{mM} \mathrm{Na}_{3} \mathrm{PO}_{4}\right.$ and $500 \mathrm{mM} \mathrm{NaCl}$, $10-400 \mathrm{mM}$ imidazole, $\mathrm{pH} 7.4$ ) at $2 \mathrm{ml} / \mathrm{min}$. The eluted samples were analyzed by Tricine-SDS-PAGE, and dialyzed against $150 \mathrm{mM} \mathrm{NaCl}$ overnight at $4^{\circ} \mathrm{C}$.

Cleavage and purification of rbE50-52. The $500 \mu \mathrm{g}$ fusion protein was treated with $250 \mathrm{U}$ SUMO protease (Invitrogen) in $10 \times$ SUMO protease buffer (2\% Igepal (NP-40), $1.5 \mathrm{M} \mathrm{NaCl}, 10 \mathrm{mM}$ DTT, $500 \mathrm{mM}$ Tris-HCl, pH 8.0) $500 \mu \mathrm{lat} 4^{\circ} \mathrm{C}$ for $15 \mathrm{~h}$. After the cleavage, proteins samples were subjected to a Ni-NTA Sepharose column to remove $6 \times$ His-tagged carrier and undigested fusion proteins. The purified rbE50-52 was analyzed by Tricine-SDS-PAGE.

Determination of the molecular weight by MS. MS analysis was carried out on AB4700 MALDI-TOF/ TOF MS (AB Company, USA). Lyophilized rbE50-52 was dissolved with solvent A (70\% acetonitrile, $0.1 \%$ trifluoroacetic acid in water $[\mathrm{v} / \mathrm{v} / \mathrm{v}]$ ), and $0.5 \mu \mathrm{l}$ samples mixed directly on the target with $1.5 \mu \mathrm{l}$ of matrix $(20 \mu \mathrm{g}$ of a-cycno- $\beta$-hydroxy-cinnamic acid/ $\mu$ in solvent $A$ ). The collected data were analyzed using Data Explorer V4.5 software (Applied Biosystems, USA).

Antimicrobial activity assay. The antimicrobial activity of rbE50-52 was tested by the agar diffusion method (cylinder plate method) using S. aureus ATCC 25923 as indicator strain. Cylinders were placed on the surface of agar inoculated with the test organism (about $1 \times 10^{7} \mathrm{CFU} / \mathrm{ml}$ ) and filled with $200 \mu \mathrm{l}$ of purified $\mathrm{rbE} 50-52(16 \mu \mathrm{g} / \mathrm{ml})$. The same volume of sodium phosphate buffer (PBS) and SUMO-bacteriocin E50-52 fusion protein were used as negative control. After incubation overnight at $37^{\circ} \mathrm{C}$, zones of growth inhibition surrounding the cylinders were measured. The minimal inhibitory concentration (MIC) and minimal bactericidal concentration (MBC) were determined by a liquid growth inhibition test using $S$. aureus ATCC 25923, E. coli K88 and L. monocytogenes IVDC C53005 as indicator strains as described by Karthikeyan (2006).

Hemolytic assay. The hemolytic property of rbE5052 was evaluated by examining hemoglobin release at $540 \mathrm{~nm}$ in suspensions of rabbit erythrocytes. Bacteriocin at different concentrations $(5,10,25,50,100 \mu \mathrm{g} / \mathrm{ml})$ was used in this experiment. The fresh rabbit blood was treated according to the method of Tian (2009). The absorption was recorded at $540 \mathrm{~nm}$ using Imark ${ }^{\mathrm{TM}}$ Microplate Reader (Bio-Rad, US). The values for $0 \%$ and $100 \%$ hemolysis were determined in $10 \mathrm{mM}$ PBS and $0.1 \%$ Triton X-100, respectively.

\section{Results}

Construction of expression vectors. The schematic representation of the bacteriocin E50-52 expression vector, containing a His-tag for affinity purification, is 


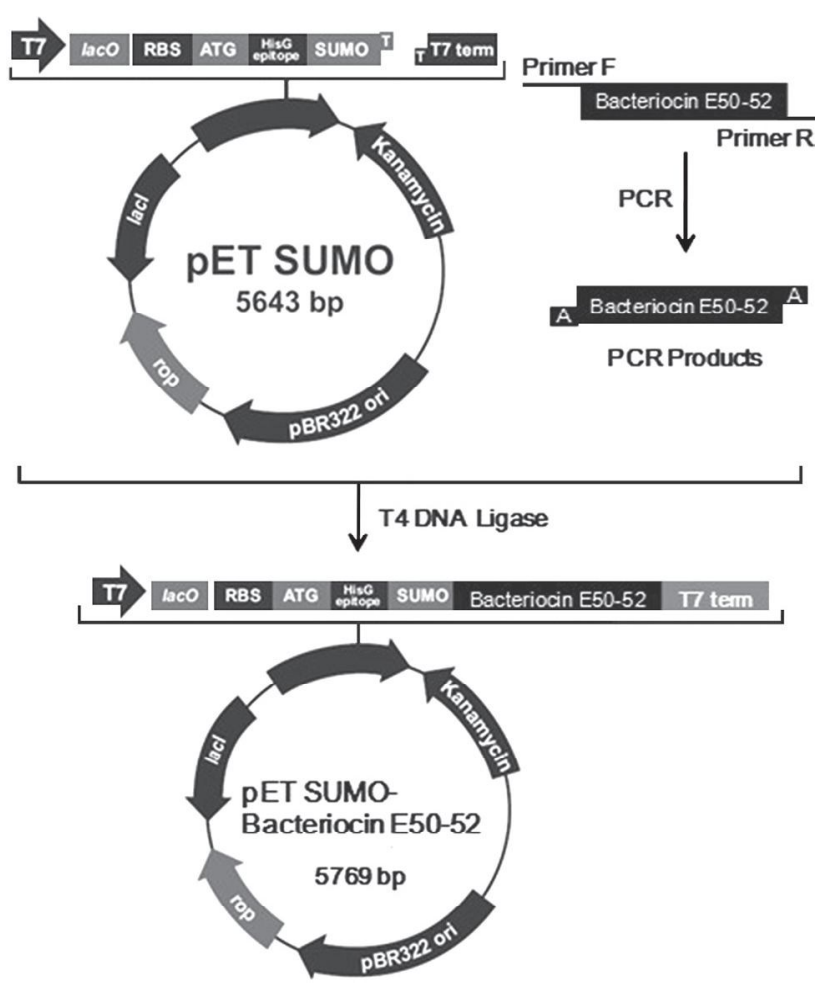

Fig. 1. Schematic representation of construction of the $\mathrm{pET}$ SUMO-bacteriocin E50-52.

After amplification, bacteriocin E50-52 gene was inserted into $\mathrm{pET}$ SUMO plasmid by TA cloning method. The plasmid with correct insert orientation was selected by sequencing.

presented in Fig. 1. The correct orientation of the insert was confirmed by PCR amplification and sequencing (data not shown).

Expression of the fusion protein. E. coli BL21 (DE3) was transformed with pET SUMO-bacteriocin E50-52 plasmid and induced as described above. After disruption by sonication, supernatant and precipitate were subject to Tricine-SDS-PAGE. As shown in Fig. 2,

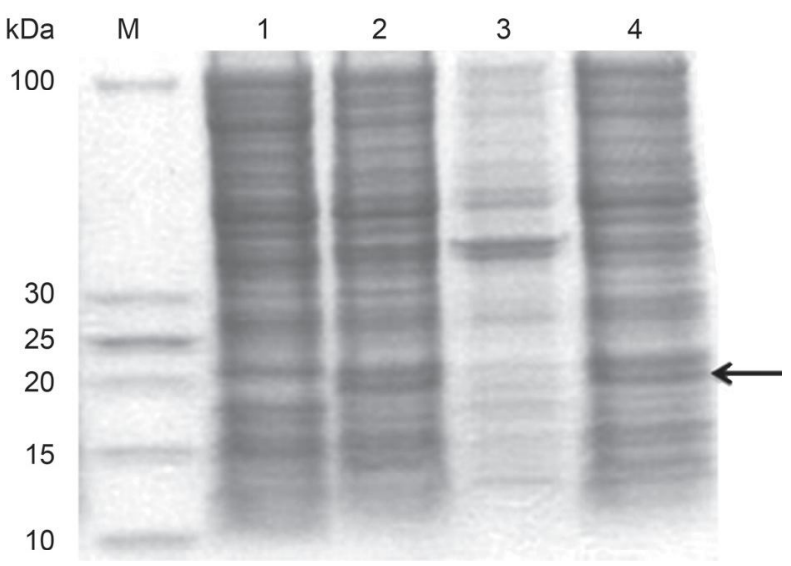

Fig. 2. Tricine-SDS-PAGE analysis of expression fusion protein.

Lane $\mathrm{M}$, protein markers; lane 1, negative control, lane 2 and 4 are soluble proteins when cultured at $1.5 \mathrm{mM} \mathrm{IPTG;}$ lane 3 is the corresponding insoluble proteins.

The arrow indicates the location of the fusion protein.

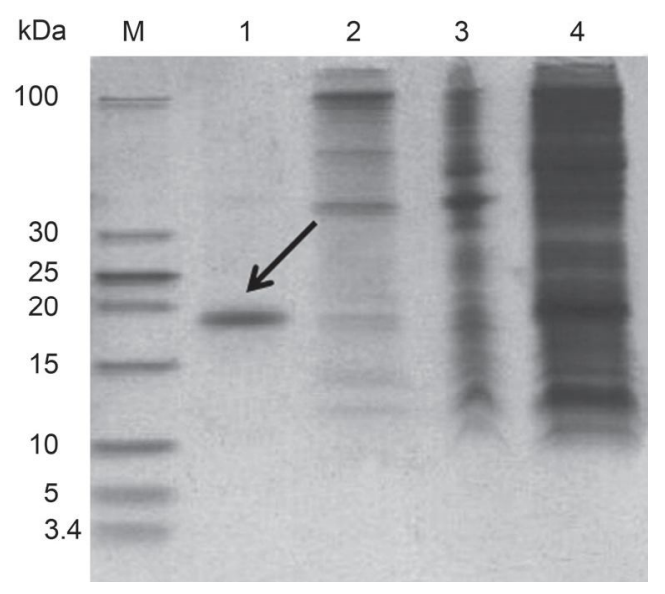

Fig. 3. Tricine-SDS-PAGE analysis of the purification of SUMO-bacteriocin E50-52 fusion protein.

The eluted fusion protein showed about $90 \%$ purity by electrophoretic analysis with Tricine-SDS-PAGE using the Bandscan software (BioMarin Pharmaceutical Inc., UK). Lane M, protein markers; lane 1, elution; lane 2, wash; lane 3, precipitation of cell lysate; lane 4, supernatant of cell lysate. The arrow indicates the purified fusion protein.

there was an obvious protein band after IPTG induction compared with non-induced cells. The result indicated that the fusion protein was efficiently produced in a soluble form in the host induced by $1.5 \mathrm{mM}$ IPTG at $37^{\circ} \mathrm{C}$.

Purification of fusion protein. The fusion protein contains polyhistidine $(6 \times \mathrm{His})$ tag at $\mathrm{N}$-terminus and can be separated efficiently using a Ni-NTA Sepharose column. Most of the proteins without His-tag were washed with washing buffer (the concentration of imidazole $<300 \mathrm{mM}$ ), and the $6 \times$ His-tagged SUMObacteriocin E.50-52 was eluted by the elution buffer (50 $\mathrm{mM} \mathrm{Na}_{3} \mathrm{PO}_{4}, 500 \mathrm{mM} \mathrm{NaCl}, 300 \mathrm{mM}$ Imidazole, $\mathrm{pH} 7.4$ ) with more than $90 \%$ purity (Fig. 3). About $117 \mathrm{mg}$ fusion protein can be obtained from 1-liter of fermentation culture.

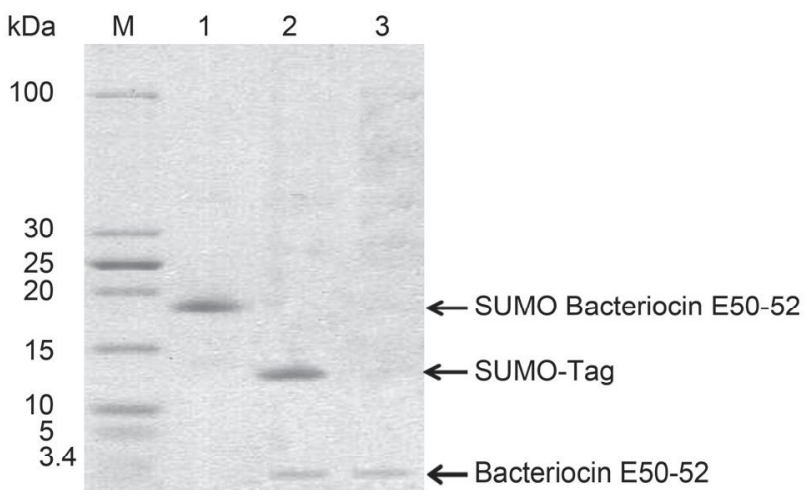

Fig. 4. Tricine-SDS-PAGE analysis of SUMO-bacteriocin E50-52 fusion protein cleaved by SUMO protease and rbE50-52 purification.

Lane M, protein markers; lane 1, purified SUMO-bacteriocin E50-52 fusion protein; lane 2, mixture of SUMO-bacteriocin E50-52 fusion protein by SUMO protease cleavage; lane 3 , purified recombinant bacteriocin E50-52 by Ni-NTA(GE). 


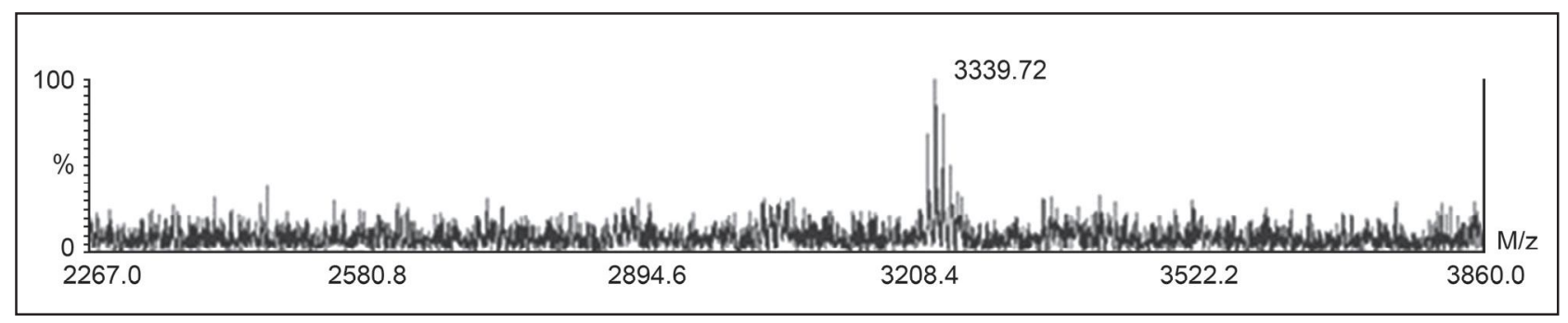

Fig. 5. MALDI-TOF/TOF MS mass spectra of purified rpE50-52.

Cleavage and purification of rbE50-52. Purified SUMO-bacteriocin E50-52 fusion protein was cleaved using SUMO protease to release the rbE50-52, and then confirmed by Tricine-SDS-PAGE. Subsequently, the mixture was loaded to a Ni-NTA Sepharose column and rbE50-52 was washed with washing buffer. As shown in Fig. 4, the fusion protein was successfully cleaved (lane 2) and rbE50-52 was purified efficiently (lane 3). About $16 \mathrm{mg}$ of purified mature rbE50-52 with more than $95 \%$ purity was obtained from 1-liter fermentation culture.

Determination of the molecular weight. The precise molecular weight of rbE50-52 determined by MS was $3339.72 \mathrm{Da}$, which is in good agreement with that of the native bacteriocin E50-52 (Fig. 5). The result suggested that the bacteriocin E50-52 was expressed correctly and the fusion protein was cleaved at expected position with no extra amino acid in the $\mathrm{N}$-terminus of recombinant peptide.

Antimicrobial activities of rbE50-52. The rbE5052 showed strong inhibitory activity against $S$. aureus ATCC 25923 (Fig. 6). The MIC and MBC against selected strains are presented in Table I. The results indicated that the rbE50-52 had similar antibacterial properties against indicator bacteria as the native bacteriocin E50-52 and was effective in killing bacteria.

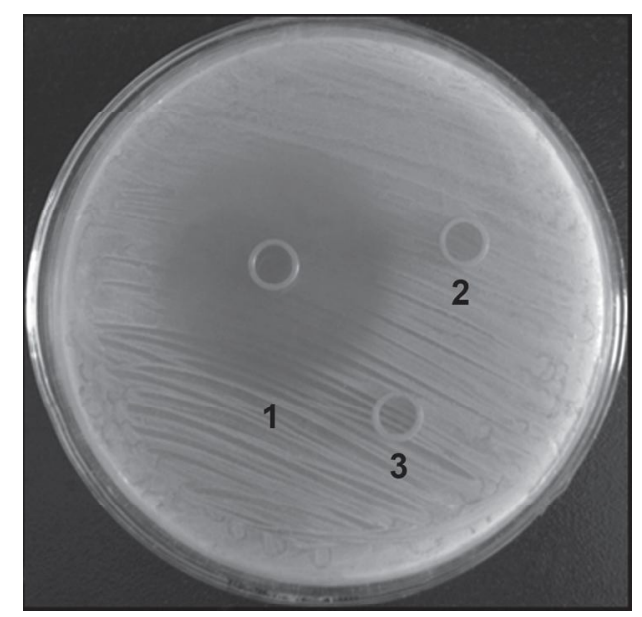

Fig. 6. The bioactivity of rbE50-52 against $S$. aureus.

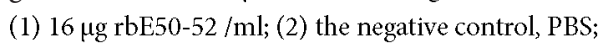
(3) $117 \mu \mathrm{g}$ SUMO-bacteriocin E50-52 fusion protein $/ \mathrm{ml}$.
Hemolytic activity. The release of hemoglobin was used to evaluate the hemolytic activity of rbE50-52. The hemolysis percentages of the peptide at concentration of $5,10,25,50,100 \mu \mathrm{g} / \mathrm{ml}$ were $0.76 \pm 0.65 \%$, $0.85 \pm 0.57 \%, 1.98 \pm 0.67 \%, 2.11 \pm 0.49 \%, 2.87 \pm 0.76 \%$, respectively.

Table I

The MIC/MBC of rbE50-52 and native bacteriocin E50-52 to selected microorganisms

\begin{tabular}{|l|c|c|}
\hline \multirow{2}{*}{\multicolumn{1}{|c|}{ Microorganisms }} & \multicolumn{2}{c|}{ MIC/MBC $(\mu \mathrm{g} / \mathrm{ml})$} \\
\cline { 2 - 3 } & rbE50-52 & $\begin{array}{c}\text { Native } \\
\text { bacteriocin } \\
\text { E50-52 }\end{array}$ \\
\hline E. coli K88 & $0.9 / 1.7$ & $<1.6 /--^{\mathrm{b}}$ \\
\hline S. aureus ATCC 25923 & $0.5 / 2$ & $0.2-0.8 /--$ \\
\hline L. monocytogenes IVDCC 53005 & $14 / 32$ & $2-32 /--$ \\
\hline
\end{tabular}

${ }^{\text {a }}$ The MIC of the native bacteriocin E50-52 against E. coli, S. aureus and L. monocytogenes were determined using many different isolates.

${ }^{\mathrm{b}}$ The MBC of native bacteriocin E50-52 against selected bacteria was not reported.

\section{Discussion}

Drug resistance among variety of microorganisms poses a potentially serious threat to public health and has caused substantial alarm and concern (Svetoch et al., 2008; 2009). It becomes urgent to develop antimicrobial compounds with new mechanisms of action and potent activity against these microorganisms. Bacteriocins can inhibit bacterial growth by disruption of target cell membrane and have a low frequency of bacteriocin-resistant mutants (Sang and Blecha, 2008; Svetoch and Stern, 2010). Bacteriocin E50-52 displayed strong inhibitory property against both Gram-positive and Gram-negative bacteria and was also effective against antibiotic-resistant bacteria isolated from hospitals in vitro test (Svetoch et al., 2008; 2009). In vivo test in broiler, oral treatment with bacteriocin E50-52 reduced both Salmonella enteritidis and Campylobacter jejuni by more than 100000 -fold in the ceca, and systemic S.enteritidis was reduced in the liver and spleen (Svetoch et al., 2008). In this work, the MBC and hemolytic activity of rbE50-52 were also measured. The 
peptide showed effective bactericidal properties against indicator strains and produced very low hemolysis against rabbit red blood cells. These characteristics of bacteriocin E50-52 make it potentially valuable as an agent for antimicrobial chemotherapy.

The low efficiency of bacteriocin purification from natural producer strains and high cost of chemical synthesis led to the exploration of heterologous expression of peptides with antimicrobial property against bacteria (Li et al., 2010). As one of the most commonly used heterologous expression host, E. coli was utilized to produce rbE50-52 in this study. Due to their high toxicity to the expression host cells and sensitivity to proteases, direct expression systems of recombinant bacteriocins are difficult to be established. So far, the best available tools for expression of these proteins are fusion protein partners, which can also increase expression level, enhance protein solubility, and simplify purification and detection of recombinant proteins (Butt et al., 2005; Esposito and Chatterjee, 2006; Li, 2009; Sun et al., 2008). Today, a number of fusion protein partners are used in the expression of proteins, such as MBP (maltose-binding protein), GST (glutathione S-transferase), TRX (thioredoxin), Nus A, Ub (ubiquitin) and SUMO. Comparative study showed that SUMO was superior to commonly used fusion partners (Marblestone et al., 2006). SUMO proteases are able to cleave SUMO tag accurately and release the recombinant protein with native N-terminus, which is prerequisite for maintaining the activity of bacteriocins ( $\mathrm{Wu}$ and Hancock, 1999; Butt et al., 2005). In the current study, the fusion protein was expressed and purified efficiently with the aid of SUMO fusion protein partners. After cleavage, the rbE50-52 with desired N-terminus was released. The recombinant peptides had similar antimicrobial properties (MIC) and molecular weight as the native bacteriocin E50-52. About $16 \mathrm{mg}$ rbE50-52 was obtained from one liter of fermentation culture, the yield of native E50-52 was not reported. Compared with isolating from natural producer stains, producing of rbE50-52 needed cheaper medium, shorter fermentation time and simpler purification procedures (Svetoch et al., 2009).

In summary, we constructed an efficient SUMO fusion system to express and purify E50-52 in E. coli. The system can produce biologically active bacteriocin E50-52 with acceptable yield, and the purification is achieved in two steps. This expression system can potentially be a powerful tool for obtaining sufficient quantities of bacteriocin E50-52 for further studies or possible application.

\section{Acknowledgements}

This work was funded by the National Natural Science Foundation of China (No. 30972124) and National High Technology Research and Development Program of China (No. 2006AA10A208).

\section{Literature}

Butt T.R., S.C. Edavettal, J.P. Hall and M.R. Mattern. 2005. SUMO fusion technology for difficult-to-express proteins. Protein Expr. Purif. 43: 1-9.

Cleveland J., T.J. Montville, I.F. Nes and M.L. Chikindas. 2001. Bacteriocins: safe, natural antimicrobials for food preservation. Int. J. Food Microbiol. 71: 1-20.

Esposito D. and D.K. Chatterjee. 2006. Enhancement of soluble protein expression through the use of fusion tags. Curr. Opin. Biotech. 17: 353-358.

Jasniewski J., C. Cailliez-Grimal, E. Gelhaye and A. RevolJunelles. 2008. Optimization of the production and purification processes of carnobacteriocins Cbn BM1 and Cbn B2 from Carnobacterium maltaromaticum $\mathrm{CP} 5$ by heterologous expression in Escherichia coli. J. Microbiol. Meth. 73: 41-48.

Jenssen H., P. Hamill and R.E.W. Hancock. 2006. Peptide Antimicrobial Agents. Clin. Microbiol. Rev. 19: 491-511.

Joerger R.D. 2003. Alternatives to antibiotics: bacteriocins, antimicrobial peptides and bacteriophages. Poultry Sci. 82: 640-647.

Johnson, E.S. 2004. Protein modification by SUMO. Annu. Rev. Biochem. 73: 355-382.

Karthikeyan M.S., D.J. Prasad, B. Poojary, K. Subrahmanya Bhat, B.S. Holla and N.S. Kumari. 2006. Synthesis and biological activity of Schiff and Mannich bases bearing 2, 4-dichloro-5-fluorophenyl moiety. Bioorgan. Med. Chem. 14: 7482-7489.

Klaenhammer T.R. 1993. Genetics of bacteriocins produced by lactic acid bacteria. FEMS Microbiol. Rev. 12: 39-85.

Li B.C., S.Q. Zhang, W.B. Dan, Y.Q. Chen and P. Cao. 2007. Expression in Escherichia coli and purification of bioactive antibacterial peptide ABP-CM4 from the Chinese silk worm, Bombyx mori. Biotechnol. Lett. 29: 1031-1036.

Li J.F., J. Zhang, R. Song, J.X. Zhang, Y. Shen and S.Q. Zhang. 2009. Production of a cytotoxic cationic antibacterial peptide in Escherichia coli using SUMO fusion partner. Appl. Microbiol. Biotechnol. 84: 383-388.

Li J.F., J. Zhang, Z. Zhang, H.W. Ma, J.X. Zhang and S.Q. Zhang. 2010. Production of Bioactive Human Beta-Defensin-4 in Escherichia coli Using SUMO Fusion Partner. Protein J. 29: 314-319.

Li Y.F. 2009. Carrier proteins for fusion expression of antimicrobial peptides in Escherichia coli. Biotechnol. Appl. Biochem. 54: 1-9.

Ma Q.S., Z.Q. Yu, B. Han, Q. Wang and R.J. Zhang. 2012. Expression and purification of lacticin Q by small ubiquitin-related modifier fusion in Escherichia coli. J. Mirobiol. 50: 326-331.

Marblestone J.G., S.C. Edavettal, Y. Lim, P. Lim, X. Zuo and T.R. Butt. 2006. Comparison of SUMO fusion technology with traditional gene fusion systems: Enhanced expression and solubility with SUMO. Protein Sci. 15: 182-189.

Morin K.M., S. Arcidiacono, R. Beckwitt and C.M. Mello. 2006. Recombinant expression of indolicidin concatamers in Escherichia coli. Appl. Microbiol. Biotechnol. 70: 698-704.

Muller S., C. Hoege, G. Pyrowolakis and S. Jentsch. 2001. SUMO, ubiquitin's mysterious cousin. Nat. Rev. Mol. Cell. Bio. 2: 202-213.

Rao X.C., S. Li, J.C. Hu, X.L. Jin, X.M. Hu, J.J. Huang, Z.J. Chen, J.M. Zhu and F.Q. Hu. 2004. A novel carrier molecule for high-level expression of peptide antibiotics in Escherichia coli. Protein Expres. Purif. 36: 11-18.

Sang Y. and F. Blecha. 2008. Antimicrobial peptides and bacteriocins: alternatives to traditional antibiotics. Anim. Health. Res. Rev. 9: 227-235.

Shen Y., H.X. Ai, R. Song, Z.N. Liang, J.F. Li and S.Q. Zhang. 2010. Expression and purification of moricin CM4 and human 
$\beta$-defensins 4 in Escherichia coli using a new technology. Microbiol. Res. 165: 713-718.

Sun Z., Z. Xia, F. Bi and J.N. Liu. 2008. Expression and purification of human urodilatin by small ubiquitin-related modifier fusion in Escherichia coli. Appl. Microbiol. Biotechnol. 78: 495-502.

Svetoch E.A., B.V. Eruslanov, V.V. Perelygin, E.V. Mitsevich, I.P. Mitsevich, V.N. Borzenkov, V.P. Levchuk, O.E. Svetoch, Y.N. Kovalev, Y.G. Stepanshin, G.R. and others. 2008. Diverse antimicrobial killing by Enterococcus faecium E50-52 bacteriocin. J. Agric. Food Chem. 56: 1942-1948.

Svetoch E.A., B.V. Eruslanov, Y.N. Kovalev, E.V. Mitsevich, I.P. Mitsevich, V.P. Levchuk, N.K. Fursova, V.V. Perelygin, Y.G. Stepanshin, M.G. Teymurasov and others. 2009. Antimicrobial Activities of Bacteriocins E50-52 and B602 Against AntibioticResistant Strains Involved in Nosocomial Infections. Probiotics \& Antimicro. Prot. 1: 136-142.
Svetoch E.A. and N.J. Stern. 2010. Bacteriocins to control Campylobacter spp. in poultry-A review. Poultry Sci. 89: 1763-1768.

Swartz J.R. 2001. Advances in Escherichia coli production of therapeutic proteins. Curr. Opin. Biotechnol. 12: 195-201.

Tian Z.G., T.T. Dong, D. Teng, Y.L. Yang and J.H. Wang. 2009. Design and characterization of novel hybrid peptides from LFB15 (W4,10), HP(2-20), and cecropin A based on structure parameters by computer-aided method. Appl. Microbiol. Biot. 82: 1097-1103.

Wei Q., Y.S. Kim, J.H. Seo, W.S. Jang, I.H. Lee and H.J. Cha. 2005. Facilitation of expression and purification of an antimicrobial peptide by fusion with baculoviral polyhedrin in Escherichia coli. Appl. Environ. Microbiol. 71: 5038-5043.

Wu M. and R.E. W. Hancock. 1999. Interaction of the Cyclic Antimicrobial Cationic Peptide Bactenecin with the Outer and Cytoplasmic Membrane. J. Biol. Chem. 274: 29-35. 